\title{
How to Make Dull Information Exciting COMMUNICATION CORNER
}

\author{
by Philip Yaffe
}

\section{Editor's Introduction}

Each "Communication Corner" essay is self-contained; however, they build on each other. For best results, before reading this essay and doing the exercise, go to the first essay "How an Ugly Duckling Became a Swan," then read each succeeding essay.

In this installment, Philip Yaffe explains how to give the reader what they want. 


\title{
How to Make Dull Information Exciting
}

\author{
COMMUNICATION CORNER
}

\section{by Philip Yaffe}

Someone once said (it may have been me): "There is no dull information, only dull writers."

We have all had the misfortune of reading very dull texts and sitting through very dull presentations. But were they inherently dull? Or were they made that way because the writer or presenter just didn't bother to do his homework?

If I asked you to read the first five pages of telephone listings, you would probably expect to die of boredom. However, if I told you somewhere in those five pages is a hidden message that could be worth $\$ 100,000$, the boredom would quickly disappear.

Why? Not because a rather uninspiring task had suddenly turned into a kind of game, although this would help. The real reason is that you would now have a clear interest to do so, and therefore a clear desire to do so.

This is a dramatic - and highly exaggerated-example of what I immodestly call "Yaffe's Law." Yaffe's Law states: If you give people what they want first, they are likely to accept anything else you want them to have. If you give them what you want first, chances are they won't accept anything at all.

By telling you that you could gain $\$ 100,000$ by reading the telephone listings, I sparked your interest. Put more bluntly, I immediately answered the question that every good writer or presenter should ask themselves: "Why the hell would anyone want to read what I am going to write or listen to what I am going to say?" Or from the audience's point of view: "What's in it for me?"

If you can't answer this question, then you might as well shut down the computer and go back to sleep, because anything you produce will be largely wasted time.

Answering this question is seldom easy; in fact, it is often very difficult. But unless you make the effort, you can hardly expect your audience to it for you. Here are a couple of examples to make the truth of Yaffe's Law more concrete. 


\section{Example 1}

[Original]

A piece of electronic equipment installed in automobiles could allow insurance companies to monitor the driving behavior of their customers.

Each time a motorist uses the car, the device will record the roads being traveled and the time of the journey, and send the information via satellite to the insurance company. With this data, the company will be able to calculate the insurance premium for each individual journey based on the relative risk of crashes on the different roads at different times of the day. The motorist will receive a monthly or quarterly "usage statement," similar to a telephone bill, itemizing the insurance cost for each use of the car.

By agreeing to the system, motorists could save hundreds of dollars on their automobile insurance.

Because of the lower risk of crashes, trips on superhighways will cost less per kilometer than on city roads and....

[Revision]

Motorists could save hundreds of dollars on their automobile insurance by allowing their driving habits to be monitored by a satellite-tracking device installed in the vehicle.

Each time a motorist uses his car, the device will record the roads being traveled and the time of the journey, and send the information to the insurance company. The company will then calculate the insurance premium based on an assessment of the relative risk of crashes on the different roads at different times of the day. Motorists will receive a monthly or quarterly "usage statement;" similar to a telephone bill, giving the insurance cost for each journey.

Because of the lower risk of crashes, trips on superhighways will cost less per kilometer than on city roads and....

Simply moving the fourth paragraph of the original to the first paragraph of the revision changes everything. Who wouldn't want to know how to save hundreds of dollars on their automobile insurance? 


\section{Example 2}

[Original]

People with mild but measurable memory problems who took the drug donepezil, trademarked Aricep, delayed the onset of Alzheimer's disease by an average of six months, a study has shown.

The research indicates that the drug works for just a short time and then stops. Still, the report is the first to find a drug therapy that delays the onset of Alzheimer's in people at high risk of the disease.

The result does not directly take researchers closer to a cure for the disease, but understanding how the drug prevents its onset could provide some important clues....

[Revision]

Donepezil, trademarked Aricep, has been shown to delay the onset of Alzheimer's disease in people at high risk, the first time any drug therapy has ever demonstrated such an effect.

A clinical study reported that the drug delayed onset of Alzheimer's by an average of six months in people with mild but measurable memory problems, a strong risk factor.

Six months of course is not very long. However, the fact that the drug worked at all is already a breakthrough, because it could provide important clues towards better prevention, treatment, and even a cure....

As you may have guessed, these two examples are taken from newspapers. This is typical of how good newspapers are written because this is typically how people want to read. But does the same technique apply outside of newspapers, and in particular in the type of writing you do?

Indeed it does. Because whatever kind of text, people still typically want to get the information in the same way:

- A clear, concise statement of what the text contains

- A clear indication (if not an actual statement) of "what's in it for me?"

When you read documents, isn't this how you would want them structured? Then this is how you should write them yourself.

For some specific writing techniques used by journalists, refer Communication Corner No. 3 "How to Improve Your Writing by Standing on Your Head." 


\section{Mathematical Magic Redux: An algebraic proof that $2=1$}

In Communication Corner No. 7, you were shown an apparent mathematical absurdity.

Start with the simple equation $a=b$. To maintain the equality, a fundamental rule of algebra says that whatever we do to the left side of the equation we must also do to the right side.

$$
\mathbf{a}=\mathbf{b}
$$

Multiply both sides by a.

$$
a^{2}=a b
$$

Subtract $b^{2}$ from both sides.

$$
a^{2}-b^{2}=a b-b^{2}
$$

Factor both sides of the equation.

$$
(a+b)(a-b)=b(a-b)
$$

Cancel the common factor $(a-b)$ from both sides of the equation.

$$
(a+b)(a-b)=b(a-b)
$$

Thus, $(\mathbf{a}+\mathbf{b}) \mathbf{=} \mathbf{b}$.

Remove the parentheses to give $\mathbf{a}+\mathbf{b}=\mathbf{b}$.

Remember the original equation $\mathbf{a}=\mathbf{b}$. Now let's substitute.

Let $\mathbf{a}=\mathbf{1}$. Then $\mathbf{b}=\mathbf{1}$.

Thus, $\mathbf{a}+\mathbf{b}=\mathbf{b}$ gives $\mathbf{1}+\mathbf{1}=\mathbf{1}$, which gives $\mathbf{2}=\mathbf{1}$.

Q.E.D.

\section{Where Is the Error?}

The error is in the step: "Cancel the common factor $a-b$ from both sides of the equation."

$$
(a+b)(a-b)=b(a-b)
$$

Canceling is something we got used to doing in school, but it is a facility, not mathematics. Canceling is a shorthand way of dividing both sides of an equation by a common factor. In other words: 


$$
\begin{array}{r}
(a+b)(a-b)=b(a-b) \\
(a-b) \quad(a-b)
\end{array}
$$

This gives $(\mathbf{a}+\mathbf{b}) \times \mathbf{1}=\mathbf{b} \times \mathbf{1}$. Removing the parentheses gives $\mathbf{a}+\mathbf{b}=\mathbf{b}$.

But this is absurd. Why? Because if $\mathbf{a}=\mathbf{b}$, then $\mathbf{a}-\mathbf{b}=\mathbf{0}$, and dividing by 0 is mathematically disallowed.

As I mentioned in the previous installment, people who immediately see the trap seem to have an approach to algebra different from those who don't. When I was in school, I remember algebra being lauded as a means of taking a specific true situation and generalizing it into a universally true situation by replacing numbers with letters.

It was deemed almost childlike to think with numbers rather than letters. But some people maintain the habit of thinking with numbers when dealing with a difficult problem. This seems to be what happens with this flawed proof. Those who can't quickly spot the error when using letters resort to using numbers. Whereas $\mathbf{a} \mathbf{-} \mathbf{b}$ does not immediately look like zero, (1 - 1) does.

To complete the story, you may be trying to remember why dividing by zero is mathematically disallowed. There are some technical explanations generally preferred by mathematicians because zero is such an unusual number. Perhaps the easiest thing to examine is what we really mean by "multiplication" and "division."

When we multiply something, we are taking that something and adding it to itself a specified number of times. For example: $2 \times 1$ is the same as adding $1+1=2$. Similarly, $3 \times 4$ is the same as adding $4+4+4=12$. And so on. Multiplying by a fraction such as $21 / 3 \times 6$ is essentially the same thing, only somewhat more complicated.

When we divide, we are in fact taking something apart. For example, if we divide 6 by 2 , we are asking how many twos do we need to make six? Of course, the answer is three, i.e. $2+2+2=6$.

But what happens with the very special number zero?

If we multiply something by zero, e.g. $0 \times 4$, we are saying add zero four times, i.e. $0+0+0+0$, which of course equals zero. The fact is, anything multiplied by zero always gives 0 zero because no matter how long the chain $0+0+0+0 \ldots$, the answer is always the same. You couldn't ask for anything easier than that!

What about division by zero?

When dividing by zero, we are in fact asking how many zeros do we need to add up to our starting number, e.g. 6 divided by $0=0+0+0+0 \ldots$ But no matter how many zeros we add together, we can never get six, or any other number. Thus, the reason dividing by zero is 
disallowed is that there can never be an answer, or as mathematicians like to say, the answer is "undefined."

In symbols, we would write $\mathbf{a} / \mathbf{0}=\mathbf{b}$. But this makes no sense because no matter what $\mathbf{a}$ is, $\mathbf{b} \times \mathbf{0}$ must always be zero. There is no other possibility.

Here is a slightly different way of looking at it. When we do division, $\mathbf{a} / \mathbf{b}=\mathbf{c}$ ( $\mathbf{a}$ divided by $\mathbf{b}$ equals $\mathbf{c}$ ), we are really asking: By what number must we multiple $\mathbf{b}$ in order to get $\mathbf{a}$, that is $\mathbf{a}=$ $\mathbf{b} \mathbf{x} \mathbf{c}$. However, if $\mathbf{b}$ is zero, then $\mathbf{b} \mathbf{x} \mathbf{c}$ must always be zero, no matter what $\mathbf{c}$ is. So there is no possible answer.

All this algebraic amusement has direct application to computers.

Because mathematicians know that dividing by zero gives undefined results, they have developed sophisticated techniques to determine the correct answer when the divisor in a complex calculation becomes zero. Basically, they divide by some small non-zero number and then take the limit as the calculation goes towards zero.

Computer scientists must be more pragmatic. Computers are built so that the arithmetic logic unit (ALU) refuses to carry out a division when the divisor is zero. It gives a "divide-by-zero error" and stops. Spreadsheets do the same thing. However, this doesn't really solve the problem. If the divisor is almost zero, the ALU will process it, but the human operator should not trust the result.

Why? The reason is the precision of the machine.

When the divisor is close to zero, the machine rounds it off to the nearest representable number. We now get a clean division. However if the representable divisor is sufficiently different from the actual divisor, the ALU will simply produce the wrong result. Since most users don't know when a "divisor almost zero" presents itself, they also don't know that they cannot trust some of the arithmetic results.

\section{HOMEWORK: Retrospective to Communication Corner No. 7}

In the previous installment (CC7), I mentioned I was once asked to write a number of case histories for one of my industrial clients. They were to be put together in a hardbound book for presentation to clients, and especially prospective clients. For this, they had to meet two very specific requirements. 
1. The company had a highly diversified range of professional products and services. Therefore, all the potential readers would not necessarily understand or even be interested in many of the case histories. It would thus be necessary to write the histories in such a way that they would attract the attention of potential readers who had no direct relationship with or understanding of the technical field each one would cover.

2. The case histories had to be as short as possible, because my client wanted the book to be highly and colorfully illustrated, with text covering no more than a single page in each double-page spread.

To meet the first requirement, we decided each text had to begin by putting the case history into a broad cultural, historical, or philosophical context potentially of interest to all reader, then move on to the special expertise my client brought to the project. To meet the second criterion, each text would have to be as short as possible, while at the same time clearly explaining any technical aspects of the project with which the wide range of potential readers might not be familiar.

I told my client that writing a rigorously short text like this is much more difficult and timeconsuming than writing a flabbily structured longer one. Indeed, if successful, the final text might appear to be self-evident, almost as if it had written itself. I had to strongly make this point to justify my fee, which otherwise might have seemed outrageous.

To demonstrate the point, I took them through a three-stage development of one such case history. The first draft was aimed essentially at digesting the background material and putting it into some kind of coherent form, i.e. "talking the text." I then wrote a shorter, more tightly structured second draft. Finally, I wrote a third draft to make the text as short as possible. If you recall, I shared the first draft of another case history, which you were asked to complete.

Remember, the objective of this homework assignment was to help you experience and remember two of the most important writing lessons you will ever learn.

Lesson 1: The most impressive thing about a well-written text is that it seems so unimpressive.

Lesson 2: Ensure that the people who have to review and approve your work fully understand why a well-written text seems so unimpressive.

Failure to properly educate your critics means that your work will not be properly valued. If you are a consultant like me, this means that you just might not get paid. 
Here is the three-draft development of the second case history. Remember, there is always more than one way to write something well. So if your second and third drafts are different from mine, don't worry. If you have met the objectives, then you will have done a good job.

\section{Draft 1 (321 words)}

Managing a railroad infrastructure has many parallels with managing a fine restaurant or a topclass hotel. Users should hardly be aware that you are there except on rare occasions when something goes wrong. And then only for the briefest amount of time needed to put it right!

However, managing a railroad infrastructure has another major concern-safety. Rail passengers have a right to expect that their train journey will be uneventful, getting them to their destination on time, and as smoothly and as comfortably as possible. With the increasing amount of rail traffic and the increasing speeds of trains, the safety aspect daily takes on ever more significance.

This is why when Infrabel, the division of the Belgian national railway charged with managing the country's rail infrastructure, decided to centralize signaling as part its attempt to upgrade reliability and safety, it turned to Enterprises $X$ (Lummen, Belgium), a recognized leader in such work. Enterprises $\mathrm{X}$ then chose to use components supplied by Engineering Solutions $\mathrm{Y}$.

The signaling upgrade is part of the Belgian railways' attempt to develop an integrated European Railway Traffic Management System. The contract with Enterprises $X$ is worth 10 million euros over five years. "The tendering criteria were particularly strict," says I.M. Anonymous, Senior Sales Engineer at Enterprises X. "Five of our staff worked for four weeks on a turnkey technical-commercial proposal."

Ensuring easy, cost-effective installation and system reliability required components of impeccable quality and a close relationship with the component supplier, which is why Enterprises $X$ turned to Engineering Solutions $Y$. "We have worked with Engineering Solutions $Y$ for many years now. Their products are of a very high standard, readily available and the working relationship is always efficient," says I.M. Anonymous.

Moreover, Engineering Solutions Y components are particularly suitable for use in harsh and industrial environment due to their resistance to vibration. This is crucial next to railway tracks where trains powerful trains constantly go thundering by.

\section{Draft 2 (238 words)}

Managing a railroad infrastructure is not unlike managing a fine restaurant or a top-class hotel. Users should hardly be aware that you are there except on rare occasions when something goes wrong, and then rapidly put right. 
However, managing a railroad infrastructure has another major concern-safety.

Passengers have a right to expect that they will get to their destination on time, and as smoothly and as comfortably as possible. With the increasing amount of rail traffic and the increasing speeds of trains, the safety aspect daily takes on ever more significance.

To achieve these objectives-reliability, comfort, safety-Infrabel, the infrastructure management division of the Belgian national railway, decided to centralize signaling. It turned to Enterprises $\mathrm{X}$, a recognized leader in such work, for the 10 million euro contract over five years. Enterprises $X$ (Lummen, Belgium) then turned to Engineering Solutions $Y$ as its preferred supplier.

Ensuring easy, cost-effective installation and system reliability required components of impeccable quality and a close relationship with the component supplier. "We have worked with Engineering Solutions $Y$ for many years now. Their products are of a very high standard, readily available and the working relationship is always efficient," says I.M. Anonymous, Senior Sales Engineer at Enterprises X.

Moreover, Engineering Solutions $Y$ components are particularly suitable for use in harsh and industrial environment, due to their resistance to vibration. This is crucial next to railway tracks where trains constantly come thundering by, I.M. Anonymous adds.

\section{Draft 3 (192 words)}

Managing a railroad infrastructure is like managing a fine restaurant or a top-class hotel. Users should hardly know you are there except on rare occasions when something goes wrong, and then is rapidly put right.

Managing a railroad infrastructure has another major concern-safely. Passengers expect that they will to their destination on time as smoothly, comfortably, and safely as possible.

To achieve these objectives Infrabel, the infrastructure management division of the Belgian national railway, decided to centralize signaling. It turned to Enterprises $X$ (Lummen, Belgium) for the 10 million euro contract over five years. They then turned to Engineering Solutions $Y$ as its preferred supplier.

Ensuring easy, cost-effective installation and system reliability required components of impeccable quality and a close relationship with the supplier. "We have worked with Engineering Solutions $Y$ for many years now. Their products are of a very high standard, readily available, and the working relationship is always efficient," says I.M. Anonymous, Senior Sales Engineer at Enterprises $\mathrm{X}$. 
Engineering Solutions $\mathrm{Y}$ components are particularly suited to harsh and industrial environment, due to their resistance to vibration. This is crucial next to railway tracks where trains constantly come thundering by.

\section{CURRENT HOMEWORK}

Here is an excerpt of an article published in an internal company newsletter. It is an excellent example of why such newsletters are generally poorly read. It clearly does not follow Yaffe's Law: If you give people what they want first, they are likely to accept anything else you want them to have. If you give them what you want first, chances are they won't accept anything at all.

Your assignment is to rewrite the text, including the headline, according to Yaffe's Law, using all the approaches and techniques you learned in previous installments, e.g. clear, concise, dense, inverted pyramid, $5 \mathrm{Ws} \& \mathrm{H}$. The names of people and companies in this exercise are fictitious, but the article is real.

Note: Key information expressed as quotations is generally more interesting and has greater impact than it does in unattributed text. Unlike real newspapers, company newsletters have the freedom to recast such information into direct quotes. However, the person for whom the quote is created must first give his or her permission for doing so. Press releases, brochures, and other company publications also often avail themselves of this freedom for the same reason-to make potentially dull information exciting.

\section{"Russian soap executives visit BubbleChem in Belgium"}

In the presence of our distribution team of Ruble \& Kopek in Russia, and Mr. Ivan Watsolvski (General Director) and Mrs. Natalia Krova (Deputy Director Science) of Gisnova, one of Russia's leading laundry detergent manufacturers, a high delegation of the most important Russian detergent association, Phomski, visited BubbleChems' facilities in Belgium from December 13-1\%.

Earlier in the year, firm contacts with Mr. Watsolvski and Mrs. Krova of Gisnova had been established when BubbleChem and Ruble \& Kopek participated in the Russian detergents conference in Perm, where our Encapsulated AntiFoam (EAF) product was presented to the Russian detergent industry.

At a time when the Russian economy seems to be rapidly recovering from the recent financial crisis, Russians are rapidly reverting to the purchase of fully automatic washing machines, which require the use and incorporation in detergent formulas of full cycle foam control -- for which purpose BubbleChem developed its patented EAF powdered foam control products. 
This product introduction attracted a lot of attention and interest. It has been sampled to many customers and is undergoing its first qualifications. Since production volumes of "low-foaming" detergents are increasing sharply, we are expecting significant new sales of EAF in Russia starting in the very near future.

Mrs. Krova was quite impressed with what she saw. "BubbleChem has great facilities and products, and we are very excited by the prospect of bringing BubbleChem's expertise as innovation to the Russian detergents market," she said

Hans Styverson, Global Marketing Leader for Household Cleaning Products at BubbleChem, said, "For us to succeed in Russia, we need the Russian voice of authority and Gisnova has built a tremendous amount of scientific experience in detergent technology and a great reputation. We are very honored that they want to work with us."

Now it's your turn. Your rewrite should give potential readers what they want first-which is certainly not a list of the names and affiliations of the people who recently visited BubbleChems' facilities. Here is a hint: Why did they visit and what does the visit mean to me as a BubbleChem employee?

\section{About the Author}

Philip Yaffe was born in Boston, Massachusetts, in 1942 and grew up in Los Angeles, where he graduated from the University of California with a degree in mathematics and physics. In his senior year, he was also editor-in-chief of the Daily Bruin, UCLA's daily student newspaper. He has more than 40 years of experience in journalism and international marketing communication. At various points in his career, he has been a teacher of journalism, a reporter/feature writer with The Wall Street Journal, an account executive with a major international press relations agency, European marketing communication director with two major international companies, and a founding partner of a specialized marketing communication agency in Brussels, Belgium, where he has lived since 1974. He is the author of more than 20 books, which can be found easily in Amazon Kindle.

DOI: $10.1145 / 3232014$ 